\title{
Performance of Missing Energy Reconstruction at the CMS detector with 2016 data
}

\author{
Leonora Vesterbacka Olsson for the CMS Collaboration* \\ ETH Zürich \\ E-mail: leonora.vesterbacka@cern.ch
}

\begin{abstract}
The performance of the missing transverse momentum reconstruction algorithms is presented using $13 \mathrm{TeV}$ pp collision data collected with the CMS detector in the beginning of 2016, corresponding to an integrated luminosity of $0.8 \mathrm{fb}^{1}$. The response and resolution for missing transverse momentum and the performance of the missing transverse momentum algorithms are measured using events with an identified $\mathrm{Z}$ boson or isolated photon. Events with anomalous missing transverse momentum are studied, and the performance of algorithms used to identify and remove those events is presented.
\end{abstract}

Fourth Annual Large Hadron Collider Physics

13-18 June 2016

Lund, Sweden

${ }^{*}$ Speaker. 


\section{Introduction}

The missing transverse momentum, $E_{\mathrm{T}}^{\text {miss }}$, is a key observable in many standard model searches as well as searches for physics beyond the standard model as it provides a way to measure particles that escape detection. The existence of for example neutrinos and hypothetical neutral weakly interacting particles can be inferred through the momentum imbalance in the plane perpendicular to the beam axis. But apart form just inferring the existence of undetected particles, the missing transverse momentum variable is sensitive to various detector effects such as imperfect resolution of the detected particles and overlapping detector signals from interactions in the previous, the same, or subsequent bunch crossing, also called pile-up. It is therefore crucial to understand the contribution from these detector effects to obtain an optimal performance of the $E_{\mathrm{T}}^{\mathrm{miss}}$.

\section{The $E_{\mathrm{T}}^{\text {miss }}$ reconstruction}

The $E_{\mathrm{T}}^{\text {miss }}$ studied is constructed as the negative vectorial sum of the momenta of all the particle flow (PF) particles in an event. The various detector effects, such as inefficiencies in the tracker, minimum energy thresholds in the calorimeters, lead to an under or over measured $E_{\mathrm{T}}^{\text {miss }}$. One way to reduce the bias on the $E_{\mathrm{T}}^{\text {miss }}$ from these effects is to correct the $p_{\mathrm{T}}$ of the jets to the particle level jet $p_{\mathrm{T}}$ according to the jet energy corrections and propagate these corrections to the $E_{\mathrm{T}}^{\text {miss }}$ according to:

$$
E_{\mathrm{T}}^{\mathrm{miss}, \mathrm{corr}}=E_{\mathrm{T}}^{\mathrm{miss}}-\Sigma_{\mathrm{jets}}\left(\vec{p}_{\mathrm{T}, \mathrm{jets}}^{\mathrm{corr}}-\vec{p}_{\mathrm{T}, \mathrm{jets}}\right)
$$

This corrected $E_{\mathrm{T}}^{\text {miss }}$ is also referred to as Type $1 E_{\mathrm{T}}^{\text {miss }}$. In Fig. 1 the distributions of the $E_{\mathrm{T}}^{\text {miss }}$ in events with $\mathrm{Z} \rightarrow \mu^{+} \mu^{-}, \mathrm{Z} \rightarrow e^{+} e^{-}$and a single photon, in data and MC. 

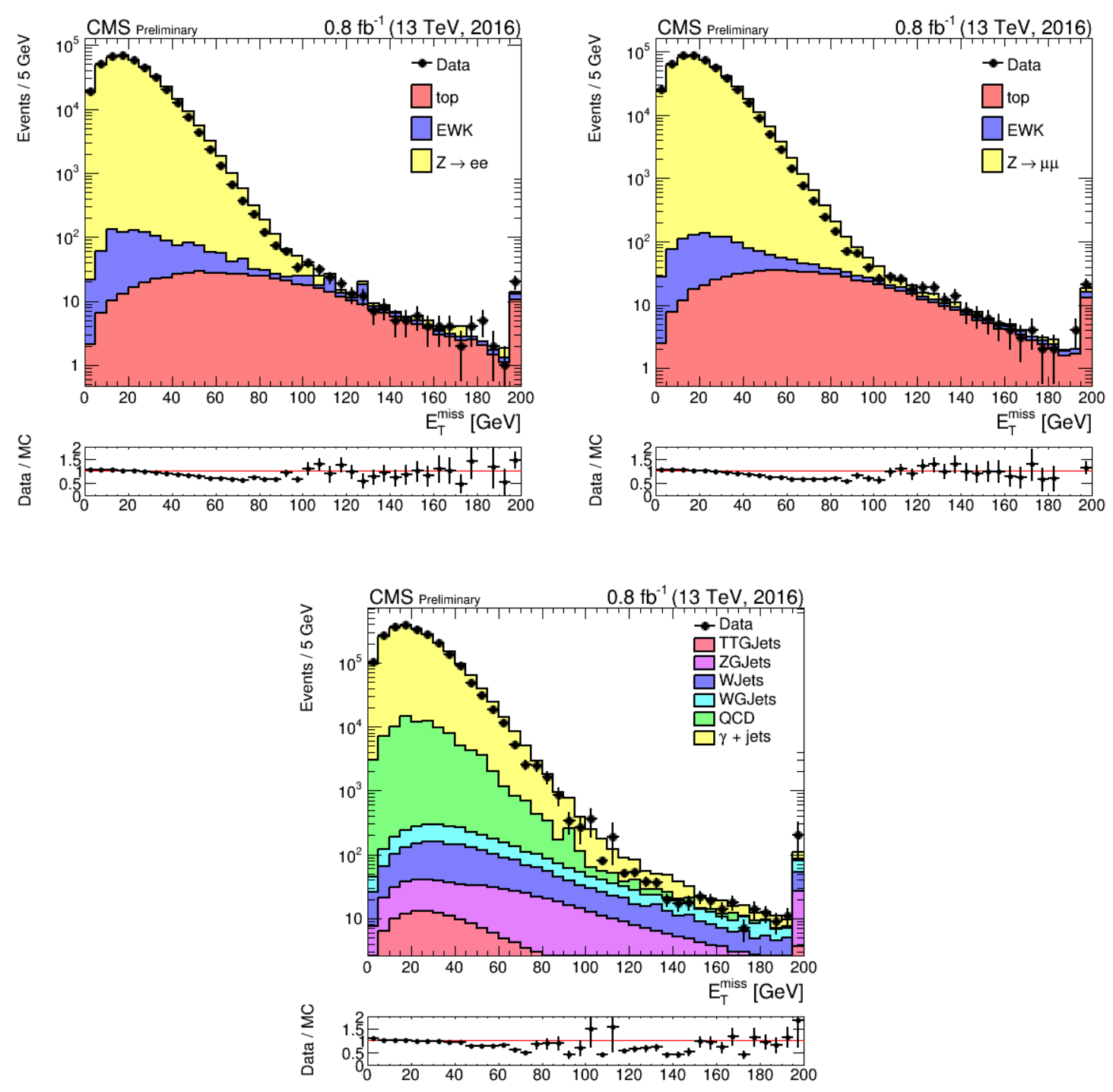

Figure 1: Distributions of Type $1 E_{\mathrm{T}}^{\text {miss }}$ in $\mathrm{Z} \rightarrow \mu^{+} \mu^{-}$(left), $\mathrm{Z} \rightarrow e^{+} e^{-}$(right), and single photon (bottom). The last bin contains overflow content. The top contribution corresponds to the top pair and single top production processes. The EWK contribution corresponds to the diboson, $\mathrm{Z} \gamma$ and $\mathrm{W} \gamma$ production processes

\section{3. $E_{\mathrm{T}}^{\text {miss }}$ response and resolution measurements}

$E_{\mathrm{T}}^{\text {miss }}$ originating only from detector effects, also called instrumental $E_{\mathrm{T}}^{\text {miss }}$, can be studied in events with two leptons originating form a $\mathrm{Z}$ boson or in events with a single photon. Both these types of events have the feature in common that the processes include no real $E_{\mathrm{T}}^{\text {miss }}$ from neutrinos, which provides ideal condition to study the pure instrumental $E_{\mathrm{T}}^{\text {miss }}$. In these events, the detector response of the hadronic system can be probed and the response and resolution of the $E_{\mathrm{T}}^{\text {miss }}$ can be measured by comparing the transverse momentum of the well measured vector boson to that of the hadronic recoil system. According to momentum conservation in the transverse plane, the 
transverse parts of the momentum of the recoil, the missing momentum and the momentum of the boson is related as follows: $\vec{u}_{T}+\vec{q}_{T}+E_{\mathrm{T}}^{\text {miss }}=0$ where $\vec{u}_{\mathrm{T}}$ is the momentum of the hadronic recoil, $\vec{q}_{\mathrm{T}}$ the momentum of the boson, illustrated in Fig. 2
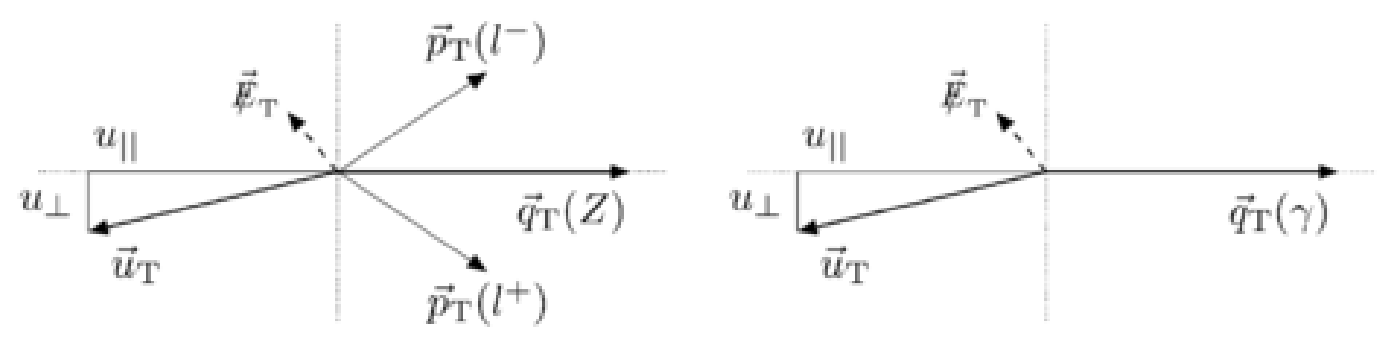

Figure 2: Illustration of $Z$ (left) and photon (right) event kinematics in the transverse plane. The vector $\vec{u}_{T}$ denotes the vectorial sum of all particles reconstructed in the event except for the two leptons from the $\mathrm{Z}$ decay (left) or the photon (right).

The hadronic recoil $\vec{u}_{T}$ can be split into its parallel and perpendicular components with respect to its axis: $u_{\|}$and $u_{\perp}$ and these components are used to study the performance of the $E_{\mathrm{T}}^{\text {miss }}$. The $E_{\mathrm{T}}^{\text {miss }}$ response is defined as the $\frac{<u_{\|}>}{<q_{\mathrm{T}}>}$ and is a variable that is related to the jet energy scale corrections. The resolution of the $E_{\mathrm{T}}^{\text {miss }}$ can be studied by the parametrization of the $u_{\|}+q_{\mathrm{T}}$ and the $u_{\perp}$ distributions by a Voigtian function. The distributions of the parallel, $u_{\|}+q_{\mathrm{T}}$, and the perpendicular, $u_{\perp}$, components of the hadronic recoil are shown in Fig. 3 in events with a $\mathrm{Z}$ boson decaying to two muons.
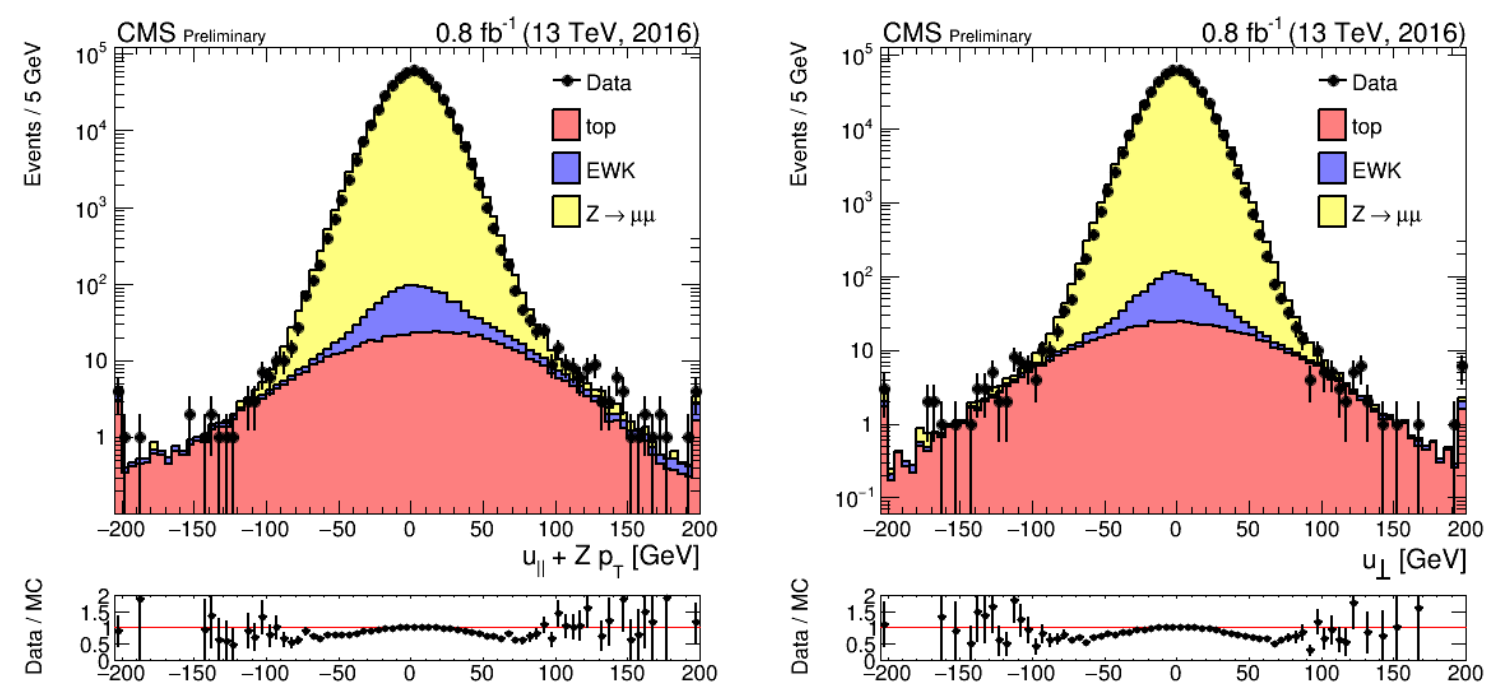

Figure 3: Distributions of the parallel, $u_{\|}+q_{\mathrm{T}}$ (left), and the perpendicular, $u_{\perp}$ (right), components of the hadronic recoil in events with a $\mathrm{Z}$ boson decaying to two muons.

The response (defined as the negative mean of the parallel component of the recoil over the boson $p_{\mathrm{T}}$ ) is shown in Fig. 4, in events with a $\mathrm{Z}$ boson decaying to electrons, muons and events 
with a single photon. The response shows an expected turn-on at low boson $p_{\mathrm{T}}$, and reaches unity at high boson $p_{\mathrm{T}}$, and the data and simulation are in good agreement for each channel.

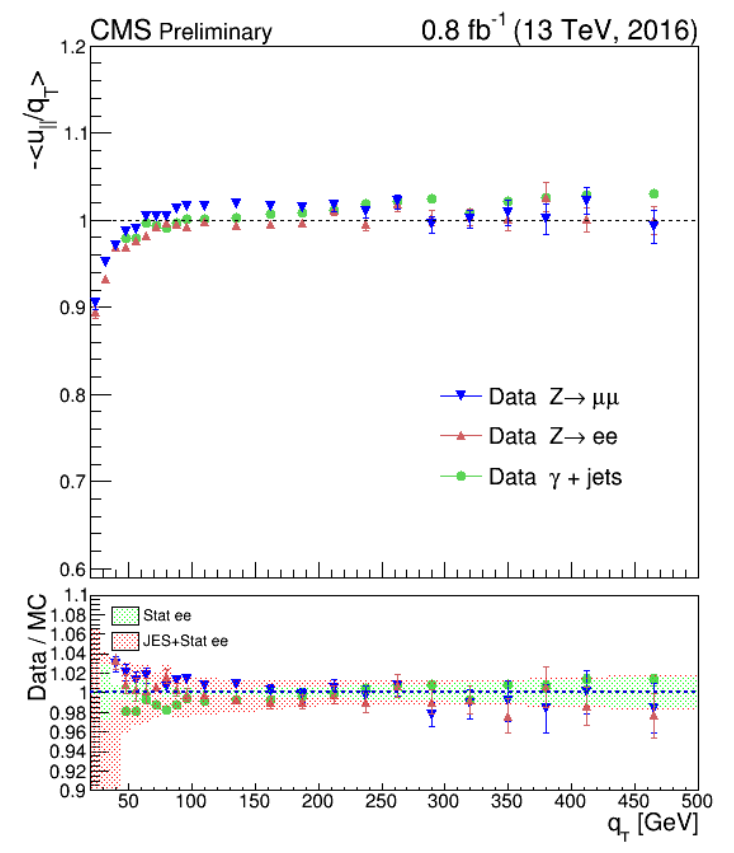

Figure 4: The response as a function of boson $p_{\mathrm{T}}$ for three different processes: blue markers correspond to events with a $\mathrm{Z}$ boson decaying to muons, red markers corresponds to a $\mathrm{Z}$ boson decaying to electrons and green markers corresponds to events with a single photon. All markers in the upper plot are events in data with the backgrounds processes subtracted, and the ratio in the bottom plot are comparisons of the data with background processes subtracted and the simulated signal background. The error bands corresponds to the statistical error and the error derived from the up/down variations of the jet energy corrections propagated to the $E_{\mathrm{T}}^{\text {miss }}$.

The resolution, defined as the parametrization of the parallel $u_{\|}+q_{\mathrm{T}}$, and the perpendicular, $u_{\perp}$ components of the hadroinc recoil by a Voigtian function as a function of the boson $p_{\mathrm{T}}$, are presented in Fig. 5. The resolution increases as expected as a function of the boson $p_{\mathrm{T}}$ and the the three channels are in good agreement between each other and the data and simulation.

\section{Anomalous $E_{\mathrm{T}}^{\mathrm{miss}}$}

The high $E_{\mathrm{T}}^{\text {miss }}$ tail is populated by anomalous events due to misreconstruction, detector noise and non-collision backgrounds. The strategy to clean these anomalous $E_{\mathrm{T}}^{\text {miss }}$ events is to apply cleaning algorithms, noise filters, and proper jet Id, and the effects of this cleaning is shown in Fig. 6 

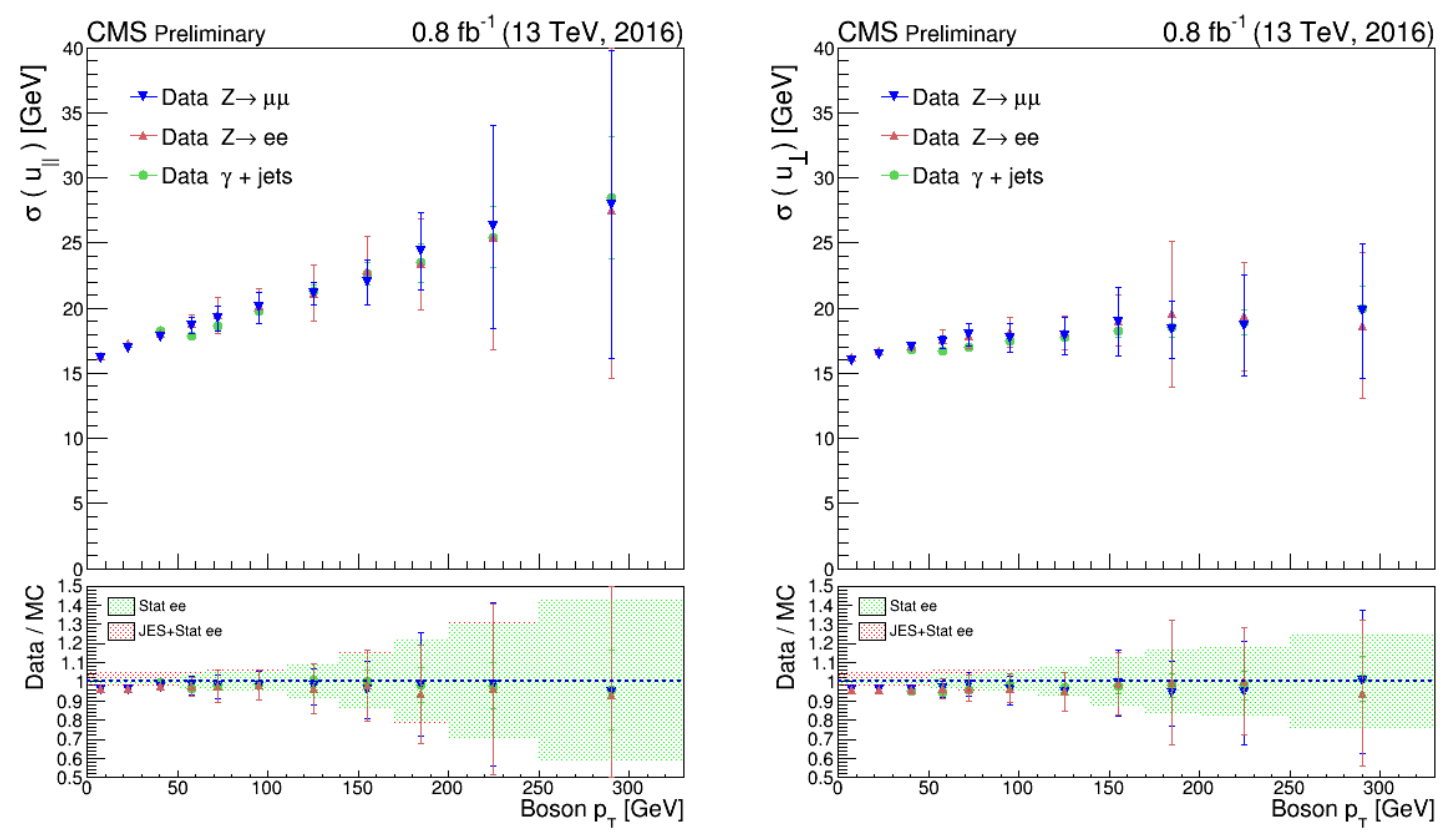

Figure 5: Distributions of the parallel, $u_{\|}+q_{\mathrm{T}}$ (left), and the perpendicular, $u_{\perp}$ (right), components of the hadronic recoil in events with a $\mathrm{Z}$ boson decaying to two muons.

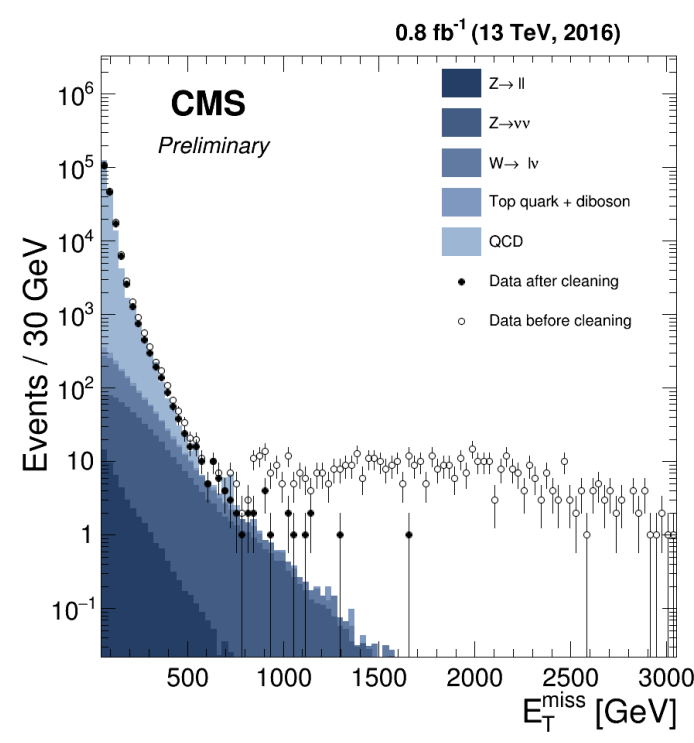

Figure 6: $E_{\mathrm{T}}^{\mathrm{miss}}$ distribution for events passing a di-jet selection without cleaning algorithms applied (open markers), with cleaning algorithms applied (filled markers), and simulated events (stacked histograms)

\section{Conclusions}

The performance of the missing transverse energy reconstruction has been presented using the first set of data collected in 2016 at a center of mass energy of $13 \mathrm{TeV}$. The response and resolution behave as expected and agree well between the three processes studied. 


\section{References}

[1] V. Khachatryan et al. [CMS Collaboration], JINST 10, no. 02, P02006 (2015) doi:10.1088/1748-0221/10/02/P02006 [arXiv:1411.0511 [physics.ins-det]]. 